\title{
The Near-IR Extinction Law
}

\author{
Joseph J. Stead and Melvin G. Hoare \\ School of Physics and Astronomy, University of Leeds, Leeds, LS2 9JT, UK
}

\begin{abstract}
We show that the power-law slope of the near-IR extinction law is significantly steeper than previously thought. Simulated colour-colour diagrams including a stellar population synthesis, realistic extinction distribution along the line-of-sight and synthesis through the filter profiles are compared to data from the UKIDSS Galactic Plane Survey. The slope of extinction with wavelength is found to be $2.14 \pm 0.05$ for total visual extinctions up to about 25 magnitudes and for a number of locations.
\end{abstract}

The typical value found in the literature for the slope of the near-IR extinction law, $\alpha$, where $A_{V} \propto \lambda^{-\alpha}$, from photometric studies of stars is $1.7 \pm 0.1$ (e.g. Draine (2003)). Here we use a new method to determine the slope of the extinction law using the new deep UKIDSS near-IR galactic plane survey data Lucas et al. (2008). J-H against H-K colourcolour diagrams for eight $60^{\prime} \times 6^{\prime}$ regions along the plane were constructed using only high-quality data. These were then compared to synthesized colour-colour data using the Besançon model of the galactic stellar population Robin et al. (2003) and a distribution of extinction along the line of sight based on those derived by Marshall et al. (2006).

Instead of adopting the usual straight reddening vector to redden the intrinsic colours of the stars, new curved reddening tracks appropriate for each stellar type were used. These reddening tracks were computed by taking model atmospheres, reddening them with a power-law of a given slope, and convolving with the UKIDSS filter profile. Tracks computed in this rigorous way are curved due to the changing effective wavelength with both spectral type and degree of reddening. These tracks are available at www.ast.leeds.ac.uk/RMS/ReddeningTracks.

To derive the slope of the extinction law synthetic colour-colour diagrams were constructed for a range of $\alpha$ values. A parameter representing the mean separation between the synthetic and observed data was calculated and minimized. Errors on the derived value of $\alpha$ were found from repeating the synthesis many times in a Monte Carlo calculation. The final value derived was $\alpha=2.14 \pm 0.05$ and it was found not to vary significantly with galactic longitude between $27 \mathrm{deg}<l<100 \mathrm{deg}$. Analysis using the same method with 2MASS data gave results which agreed within the errors. We believe our slope is larger than the previously derived values due to the choice of filter wavelength used to convert observed colour excess ratios to power-law slopes. Details of this work can be found in Stead \& Hoare (2009). In future this will be applied to dereddening individual members of young clusters associated with massive young stellar objects from the RMS survey (see Lumsden et al., this volume).

\section{References}

Draine, B. T. 2003, ARAA, 41, 241

Lucas, P. W. et al. 2008, MNRAS, 391, 136

Marshall, D. J., Robin, A. C., Reylé, C., Schultheis, M., \& Picaud, S. 2006, A $\mathscr{E} A, 453,635$

Robin, A. C., Reylé, C., Derrière, S., \& Picaud, S. 2003, A\& A, 409, 523

Stead, J. J. \& Hoare, M. G. 2009, MNRAS, 400, 731 